\title{
A CONTRIBUIÇÃO DOS PROGRAMAS DE EDUCAÇÃO EMPREENDEDORA PARA O EGRESSO: UM CAMINHAR PELAS PUBLICAÇÕES SOBRE O TEMA
}

Flávia Alexandrina Coelho Marcos ${ }^{1}$

Sandra Regina Holanda Mariano ${ }^{1}$ 


\section{A CONTRIBUIÇÃO DOS PROGRAMAS DE EDUCAÇÃO EMPREENDEDORA PARA O EGRESSO: UM CAMINHAR PELAS PUBLICAÇÕES SOBRE O TEMA}

Resumo: Este artigo objetiva compreender a contribuição da educação empreendedora para o futuro profissional dos egressos de um curso de formação em empreendedorismo. A metodologia utilizada foi uma pesquisa bibliométrica e análise de conteúdo dos artigos publicados na base de dados Web of Science e Scopus, no período de 2000 a 2018. Foram identificados 18 artigos de interesse, que foram lidos e organizados de acordo com autores, citações, países que mais publicam na área, assuntos, abordagem e teorias usadas para verificar o impacto da EE. Os achados sugerem que programas de educação empreendedora ampliam a perspectiva da escolha de carreira, ajudam na conquista de um emprego, contribuem para a redução da pobreza e apoiam o desenvolvimento de regiões. Algumas limitações foram apontadas como a necessidade de formar profissionais para trabalhar a $\mathrm{EE} \mathrm{e}$ a carência de teorias que ofereçam suporte mais robusto para o desenvolvimento da área de empreendedorismo.

Palavras chave:

Educação. Empreendedorismo. Carreira. Impacto. 


\section{Introdução}

No Brasil, desde o início da década de 1990 o estudo do empreendedorismo vem ganhando cada vez mais importância (MORAES, 2016). Para autores clássicos da área de administração como Peter Drucker, o empreendedorismo pode ser definido como uma forma de ser e de se comportar em sociedade (DRUCKER, 1998). Ser empreendedor é mais do que abrir uma empresa, é entender o seu papel na sociedade de impulsionador do motor econômico e social. Para Mariano e Mayer (2012), "ser empreendedor ou empreendedora é tornar-se um agente de transformação da própria vida e da comunidade, construindo algo de valor para a sociedade" (MARIANO; MAYER, 2012, p. 56).

A educação empreendedora fomenta atitudes que estimulam o desenvolvimento econômico e social. Esta educação pode ser desenvolvida, ou seja, as pessoas podem ser educadas para empreender, ampliando suas possibilidades de realização profissional, inclusive, a partir da abertura do próprio negócio, sendo estes empreendedores empresariais. Outros tipos de empreendedores são os empreendedores sociais, que tem como objetivo a mudança da realidade social. Há, também, os empreendedores corporativos ou intraempreendedores, que atuam dentro de uma empresa contribuindo com algo criativo, gerando riqueza e valor para a sociedade, dotado de atitudes e características empreendedoras (FILION, 2004; MARIANO, 2012).

O impulso dos indivíduos para o crescimento e a inovação está relacionado com a educação para o empreendedorismo (LANDSTRÖM, 2012). A geração de ideias novas é importante para o desenvolvimento dos setores da sociedade (MOBERG, 2014). Neste sentido, é possível educar para o empreendedorismo desde a educação básica e desenvolver a mentalidade empreendedora (ROSENDAHL, 2012).

Os esforços para prover educação empreendedora (EE) vêm crescendo no mundo inteiro e nos diversos segmentos de ensino, desde a educação básica até o nível universitário (FAYOLLE, 2013). Os programas voltados para a educação empreendedora (PEE) impactam nas intenções empreendedoras, que se relacionam ao estado de espírito ou a propensão do indivíduo para empreender. Os PEEs contribuem para o desenvolvimento do comportamento empreendedor, além de contribuir para o desenvolvimento de competências importantes tais como a persistência e a autoeficácia (FAYOLLE, 2015).

Uma temática relevante para a empreendedorismo é a avaliação da eficácia dos PEE que podem ser observados pelos conteúdos, design e entrega (ZHAO, 2015). Uma das teorias utilizadas para verificar a contribuição da EE é a Teoria do Comportamento Planejado (TCP). A TCP verifica as relações atitudes-comportamento e leva em consideração as variáveis externa e interna como influenciadoras da ação humana (AJZEN, 1985).

Assim como a educação empreendedora deve levar em consideração aspectos culturais e econômicos, a escolha da carreira é influenciada por esses aspectos. Carreira é uma "profissão ou percurso profissional", segundo o dicionário Aurélio. A carreira está relacionada, também, ao aspecto político (CHANLAT, 1996). Sendo assim, o desenvolvimento de competências empreendedoras pode auxiliar na projeção de carreira. Uma visão baseada em competências auxilia no desenvolvimento do percurso profissional. A rede de contatos, desenvolvida na visão empreendedora, e outras competências como as pessoais têm relação com a projeção de carreira (TAMS, 2006).

O presente estudo visa compreender e identificar a contribuição da EE para egressos de curso de formação em empreendedorismo para a trajetória profissional e o futuro dos estudantes. Desta forma, será observado como a temática tem sido abordada no campo de 
pesquisa das ciências sociais aplicadas. Portanto, este artigo busca responder ao seguinte questionamento: Qual é a contribuição de um PEE para os egressos dos cursos?

Para fins deste estudo, foi realizada uma pesquisa bibliométrica nas bases de dados Web of Science e Scopus, seguidas de leitura dos resumos e download dos artigos para posterior análise de conteúdo. Os artigos foram organizados de acordo com autores, citações e países que mais publicam na área. Além dessa introdução, o trabalho está dividido em 4 seções: metodologia, análise de resultados, considerações finais e referências bibliográficas.

\section{Metodologia da pesquisa}

A presente pesquisa trata de um estudo de natureza qualitativo e quantitativo com abordagem exploratória e de análise de conteúdo. Para entender as publicações sobre a contribuição da educação empreendedora ou educação para o empreendedorismo para os egressos de um PEE foi realizada uma análise bibliométrica, também conhecida como "bibliografia estatística". Utilizou-se indicadores de produção científica, demonstrado pelo número de citações de um artigo. A proposta é a geração de uma análise da área através de um apanhado geral das publicações.

A pesquisa ocorreu na base de dados da Web of Science e Scopus, abrangendo o período de janeiro de 2000 a janeiro de 2018. As bases de dados são internacionais e a escolha ocorreu devido às bases possuírem cobertura em revistas de alto impacto (MARCELO, 2013).

A pesquisa foi realizada no dia 16 de janeiro de 2018, utilizando os termos: "Entrepren* Education and professional career", "Entrepren* education AND trajectory professional", "Entrepren* Education and for college adults", "Entrepren* education and impact for program participants". Foi realizada uma leitura das palavras chaves e dos resumos, utilizando a técnica de análise conteúdo. Para Bardin (2006), uma análise de conteúdo deve esgotar o assunto, obter amostras que representam o universo, ter homogeneidade dos dados, buscando os temas que sejam os mesmos e ter pertinência na escolha dos documentos com os objetivos da pesquisa.

Para este estudo foi realizado o seguinte: (a) definição das palavras chaves para a busca na base de dados; (b) realização da busca dos artigos; (c) download dos artigos para confrontar algumas informações obtidas nos resumos; (d) Criação de uma planilha no excel relacionando os artigos, autores, país de afiliação, número de publicações; (e) Categorização dos artigos por teorias e assuntos abordados; (f) Seleção dos artigos através de uma análise de conteúdo; (g) leitura dos artigos de interesse; (h) Análise do conteúdo dos artigos de interesse.

\section{Análise dos Resultados}

A presente pesquisa ocorreu na base de dados Web of Science e Scopus. Foram pesquisadas as combinações dos termos a seguir e encontrados os resultados relacionados. Conforme demonstrado na tabela 1 e 2, observou-se um maior quantitativo de artigos na busca com os termos "Entrepren* Education and professional career".

\begin{tabular}{c|c}
\hline Busca de Dados & Resultado p/ Registro \\
\hline "Entrepren* Education and professional career" & 100 \\
\hline "Entrepren* education AND trajectory professional”, & 7 \\
\hline "Entrepren* Education and for college adults" & 6 \\
\hline "Entrepren* education and impact for program participants & 41 \\
\hline Total & $\mathbf{1 5 4}$ \\
\hline
\end{tabular}

Tabela 1: Histórico de pesquisas na Base de dados Web of Science

Fonte: Dados da Pesquisa 


\begin{tabular}{l|c}
\hline Busca de Dados & Resultado p/ Registro \\
\hline "Entrepren* Education and professional career" & 188 \\
\hline "Entrepren* education AND trajectory professional”, & 10 \\
\hline "Entrepren* Education and for college adults” & 20 \\
\hline "Entrepren* education and impact for program participants & 62 \\
\hline Total & $\mathbf{2 8 0}$ \\
\hline
\end{tabular}

Tabela 2: Histórico de pesquisas na Base de dados Scopus

Fonte: Dados da Pesquisa

Após a triagem dos artigos realizada a partir da análise dos resumos que buscava identificar artigos que abordassem a contribuição dos PEE para os egressos dos cursos, chegou-se ao resultado apresentado na Figura 1 que mostra a evolução anual do número de publicações.

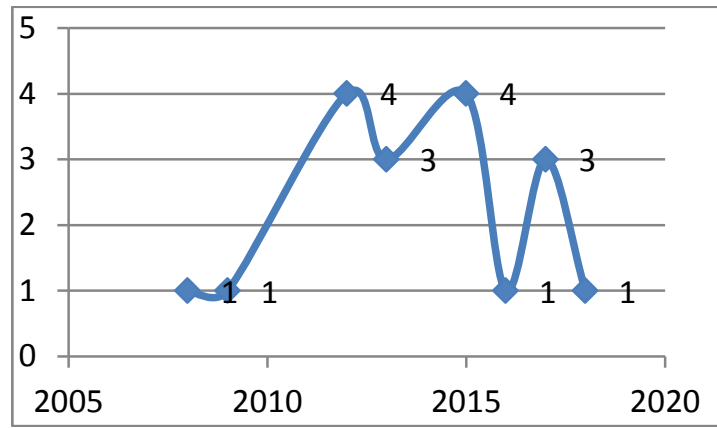

Figura 1 - Evolução anual das publicações após triagem dos artigos

Fonte: Dados da Pesquisa

Percebe-se que há variações constantes no número de publicações na área. Nos primeiros anos pesquisados, 2000 a 2007 não houve publicação relacionando educação empreendedora a carreira ou ao desenvolvimento profissional. $\mathrm{O}$ tema passou a ser tratado inicialmente em 2012. Os anos de 2015 e 2017 apresentaram a maior quantidade de artigos publicados. Observou-se que no primeiro mês de 2018 foram identificados dois artigos, mais do que o ano inteiro de 2008, 2009 e 2016. A evolução anual das publicações após triagem dos artigos retrata que há um baixo quantitativo de publicações sobre a temática.

A Tabela 3 apresenta os países que publicaram sobre a contribuição da EE para os egressos de um curso de formação em empreendedorismo. Nela está apresentado o ano da primeira publicação dos artigos.

\begin{tabular}{c|c|c}
\hline País da publicação & No. Artigos & $\begin{array}{c}\text { Ano de } \\
\text { Publicação do 1o. }\end{array}$ \\
\hline EUA & 5 & 2012 \\
\hline REINO UNIDO & 3 & 2012 \\
\hline BÉLGICA & 2 & 2009 \\
\hline PORTUGAL & 2 & 2012 \\
\hline FRANÇA & 1 & 2017 \\
\hline ITÁLIA & 1 & 2017 \\
\hline IRAN / HOLANDA & 1 & 2016 \\
\hline ESPANHA & 1 & 2015 \\
\hline GRÉCIA & 1 & 2008 \\
\hline TUNÍSIA & 1 & 2013 \\
\hline
\end{tabular}

Tabela 3 - Publicação por países e início da pesqui

Fonte: Dados da Pesquisa 
A tabela 3 apresenta uma pulverização na publicação dos artigos sobre a temática, com apenas uma publicação de cada país, com exceção dos Estados Unidos da América (EUA) que foi o país de origem de cinco publicações. A EE floresceu nos EUA, onde as escolas são estimuladas às atividades empreendedoras e, inclusive, são classificadas de acordo com o nível de EE (LOPES, 2017). O Reino Unido, Bélgica e Portugal são origem de duas publicações cada um. A partir de 2003, a educação empreendedora ganhou importância no âmbito da União Européia que passou a estimular de forma mais direta o empreendedorismo, inclusive, para estratégias e políticas recomendadas aos Estados membros (LOPES, 2017).

A tabela 4 apresenta as publicações e suas respectivas citações nas duas bases de dados: Web of Science e Scopus.

Tabela 4 - As publicações e número de citações organizado por ano de publicação Fonte: Desenvolvido pelo autor (2018)

\begin{tabular}{|c|c|c|c|c|c|}
\hline $\begin{array}{l}\text { Cita- } \\
\text { çôes } \\
\text { WoS }\end{array}$ & \begin{tabular}{|c|}
$\begin{array}{c}\text { Cita- } \\
\text { cões } \\
\text { Scopus }\end{array}$ \\
\end{tabular} & Autor & Ano & Título do artigo & Onde foi publicado \\
\hline & 0 & KOLADE, O. & 2018 & $\begin{array}{l}\text { Venturing under fire: } \\
\text { Entrepreneurship education. } \\
\text { venture creation, and poverty } \\
\text { reduction in conflict-ridden } \\
\text { Maiduguri, Nigeria }\end{array}$ & $\begin{array}{l}\text { EDUCATION AND TRAINING, 22, } \\
2018\end{array}$ \\
\hline 0 & & KOVESI, K. & 2017 & $\begin{array}{l}\text { The changing face of } \\
\text { entrepreneurship education for } \\
\text { engineering students in France }\end{array}$ & $\begin{array}{l}\text { CONFERÊNCIA: 8TH IEEE } \\
\text { GLOBAL ENGINEERING } \\
\text { EDUCATION GREECE (EDUCON) } \\
\text { ATHENS, GREECE, APR 25-28 }\end{array}$ \\
\hline \multirow[t]{2}{*}{0} & & $\begin{array}{l}\text { PEDRINI, M.; } \\
\text { LANGULLA, V.; } \\
\text { MOLTENI, M. }\end{array}$ & 2017 & \begin{tabular}{|} 
Do entrepreneurial education \\
programs impact the \\
antecedents of entrepreneurial \\
intention? An analysis of an \\
entrepreneurship MBA in Ghana \\
\end{tabular} & $\begin{array}{l}\text { JOURNAL OF ENTERPRISING } \\
\text { COMMUNITIES-PEOPLE AND } \\
\text { PLACES OF GLOBAL ECONOMY } \\
\text { Vol.: } 11 \text { Ed·: } 3 \text { Edição especial: SI } \\
\text { Pág.: } 373-392 \\
\end{array}$ \\
\hline & 0 & $\begin{array}{l}\text { ARPIAINEN, R.; } \\
\text { KURCZEWSKA, A. }\end{array}$ & 2017 & \begin{tabular}{|c|} 
Learning risk-taking and \\
coping with uncertainty through \\
experiential, team based- \\
entrepreneurship education \\
\end{tabular} & $\begin{array}{l}\text { INDUSTRY AND HIGHER } \\
\text { EDUCATIONVOLUME, Vol. 31, } \\
\text { Issue 3, } 1 \text { June 2017, Pag. 143-155 }\end{array}$ \\
\hline 24 & & $\begin{array}{c}\text { KARIMI, S.; } \\
\text { BIEMANS, H. J. } \\
\text { A.; LANS, T.; et al. }\end{array}$ & 2016 & $\begin{array}{c}\text { The Impact of } \\
\text { Entrepreneurship Education: A } \\
\text { Study of Iranian Students' } \\
\text { Entrepreneurial Intentions and } \\
\text { Opportunity Identification } \\
\end{array}$ & $\begin{array}{l}\text { JORNAL OF SMALL BUSINESS } \\
\text { MANAGEMENT Volume: } 54 \text { Ed. : } 1 \\
\text { Pág.: 187-209 } \\
\end{array}$ \\
\hline 1 & & $\begin{array}{l}\text { DIAZ-GARCIA, } \\
\text { C.; SAEZ,- } \\
\text { MARTINEZ, F.; } \\
\text { JIMENEZ- } \\
\text { MORENO, J. } \\
\end{array}$ & 2015 & $\begin{array}{l}\text { Assessing the impact of the } \\
\text { "Entrepreneurs" education } \\
\text { programme on participants' } \\
\text { entrepreneurial intentions }\end{array}$ & $\begin{array}{c}\text { RUSC-UNIVERSITIES AND } \\
\text { KNOWLEDGE SOCIETY } \\
\text { JOURNAL Vol .: } 12 \text { Ed.: } 3 \text { Pág.: 17-31 }\end{array}$ \\
\hline 1 & 1 & $\begin{array}{l}\text { KEENA, L.; } \\
\text { SIMMONS, C. }\end{array}$ & 2015 & $\begin{array}{l}\text { Rethink, Reform, Reenter: An } \\
\text { Entrepreneurial Approach to } \\
\text { Prison Programming }\end{array}$ & $\begin{array}{l}\text { INTERNATIONAL JOURNAL OF } \\
\text { OFFENDER THERAPY AND } \\
\text { COMPARATIVE CRIMINOLOGY } \\
\text { Volume: } 59 \quad \text { Ed .: } 8 \quad \text { Pág.: } 837-854\end{array}$ \\
\hline 64 & 69 & $\begin{array}{l}\text { FAYOLLE, A.; } \\
\text { GAILLY, B. }\end{array}$ & 2015 & $\begin{array}{c}\text { The Impact of Entrepreneurship } \\
\text { Education on Entrepreneurial } \\
\text { Attitudes and Intention: } \\
\text { Hysteresis and Persistence }\end{array}$ & $\begin{array}{l}\text { JOURNAL OF SMALL BUSINESS } \\
\text { MANAGEMENT Volume: } 53 \text { Ed .: } \\
1 \text { Pág.: } 75-93\end{array}$ \\
\hline
\end{tabular}




\begin{tabular}{|c|c|c|c|c|c|}
\hline $\begin{array}{l}\text { Cita- } \\
\text { ções } \\
\text { WoS }\end{array}$ & $\begin{array}{c}\text { Cita- } \\
\text { ções } \\
\text { Scopus }\end{array}$ & Autor & Ano & Título do artigo & Onde foi publicado \\
\hline & 1 & $\begin{array}{l}\text { CARVALHO, } \\
\text { L.; COSTA, T.; } \\
\text { MARES, P. }\end{array}$ & 2015 & $\begin{array}{l}\text { A success story in a partnership } \\
\text { programme for } \\
\text { entrepreneurship education: } \\
\text { Outlook of students perceptions } \\
\text { towards entrepreneurship } \\
\end{array}$ & $\begin{array}{l}\text { INTERNATIONAL JOURNAL OF } \\
\text { MANAGEMENT IN EDUCATION, } \\
\text { Vol.9, Issue 4, 2015, Pag. 444-465 }\end{array}$ \\
\hline 2 & & $\begin{array}{l}\text { NASHR, B. K., } \\
\text { BOUJELBENE } \\
, \mathrm{Y} .\end{array}$ & 2013 & $\begin{array}{l}\text { Assessing the impact of } \\
\text { entrepreneurship education }\end{array}$ & $\begin{array}{c}\text { CONFERÊNCIA: 2ND WORLD } \\
\text { CONFERENCE ON BUSINESS, } \\
\text { Economics and Management (BEM) } \\
\text { Local: Antalya, TURKEY, APR 25-28 }\end{array}$ \\
\hline 0 & & $\begin{array}{l}\text { DUVAL - } \\
\text { COUETIL, N.; } \\
\text { WHEADON, J. }\end{array}$ & 2013 & $\begin{array}{c}\text { The Value of Entrepreneurship } \\
\text { to Recent Engineering } \\
\text { Graduates: A Qualitative } \\
\text { Perspective }\end{array}$ & $\begin{array}{l}\text { CONFERENCIA: 43RD ANNUAL } \\
\text { FRONTIERS IN EDUCATION } \\
\text { CONFERENCE (FIE) LOCAL: } \\
\text { UNIV OKLAHOMA, Coll Engn, } \\
\text { Oklahoma City, OK Data: OCT } 23-25\end{array}$ \\
\hline 0 & & $\begin{array}{l}\text { MASSI, L.; } \\
\text { GEORGIOPO } \\
\text { ULOS, M.; } \\
\text { YOUNG, C. } \\
\text { Y.; Et. al. }\end{array}$ & 2013 & $\begin{array}{l}\text { Internships and Undergraduate } \\
\text { Research: Impact, Support, and } \\
\text { Institutionalization of an NSF } \\
\text { S-STEM Program through } \\
\text { Partnerships with Industry and } \\
\text { Funding from Federal and } \\
\text { Local Workforce Agencies }\end{array}$ & $\begin{array}{c}\text { CONFERÊNCIA: ASEE ANNUAL } \\
\text { CONFERENCE Local: Atlanta, GA } \\
\text { Data: JUN 23-26 }\end{array}$ \\
\hline 0 & & $\begin{array}{l}\text { CARVALHO, } \\
\text { M. I.; } \\
\text { SIMOES, J.; } \\
\text { SAMAGAIO, } \\
\text { A.; et. al. }\end{array}$ & 2012 & $\begin{array}{l}\text { Enterprise Potential of } \\
\text { Portuguese Students Fostered } \\
\text { by an Entrepreneurship } \\
\text { Education Program }\end{array}$ & $\begin{array}{l}\text { CONFERENCIA: 7TH EUROPEAN } \\
\text { CONFERENCE ON INNOVATION } \\
\text { AND ENTREPRENEURSHIP } \\
\text { (ECIE) Local: Inst Politecnico } \\
\text { Santarem, Escola Super Gestao \& } \\
\text { Tecnologia, PORTUGAL, SEP } 20-21 \\
\end{array}$ \\
\hline & 46 & $\begin{array}{l}\text { DUVAL- } \\
\text { COUETIL, } \\
\text { N.; REED- } \\
\text { RHOADS, T.; } \\
\text { HAGHIGHI,S. }\end{array}$ & 2012 & $\begin{array}{l}\text { Engineering students and } \\
\text { entrepreneurship education: } \\
\text { Involvement, attitudes and } \\
\text { outcomes }\end{array}$ & $\begin{array}{l}\text { International Journal of Engineering } \\
\text { Education Vol. 28, No. 2, pp. } 425- \\
435\end{array}$ \\
\hline \multirow[t]{3}{*}{17} & 22 & $\begin{array}{l}\text { GORDON, I.; } \\
\text { HAMILTON, } \\
\text { E.; JACK, S. }\end{array}$ & 2012 & $\begin{array}{c}\text { A study of a university-led } \\
\text { entrepreneurship education } \\
\text { programme for small business } \\
\text { owner/managers }\end{array}$ & $\begin{array}{c}\text { ENTREPRENEURSHIP AND } \\
\text { REGIONAL DEVELOPMENT } \\
\text { Volume 24, Issue 9-10, December } \\
\text { 2012, Pag. 767-805 } \\
\end{array}$ \\
\hline & 17 & $\begin{array}{l}\text { ELMUTI, D.; } \\
\text { KHOURY, G.; } \\
\text { OMRAN, O. }\end{array}$ & 2012 & $\begin{array}{c}\text { Does entrepreneurship } \\
\text { education have a role in } \\
\text { developing entrepreneurial } \\
\text { skills and ventures' } \\
\text { effectiveness? }\end{array}$ & $\begin{array}{c}\text { JOURNAL OF } \\
\text { ENTREPRENEURSHIP } \\
\text { EDUCATION Volum e 15, 2012, } \\
\text { Pages } 83-98\end{array}$ \\
\hline & 10 & $\begin{array}{l}\text { FAYOLLE, A.; } \\
\text { GAILLY, B. }\end{array}$ & 2009 & $\begin{array}{c}\text { Évaluation d'une formation en } \\
\text { entrepreneuriat: Prédispositions } \\
\text { et impact sur l'intention } \\
\text { d'entreprendre } \\
\end{array}$ & $\begin{array}{l}\text { MANAGEMENT OPEN ACCESS } \\
\text { Vol. 12, Issue 3, 2009, Pag. 175-203 }\end{array}$ \\
\hline 2 & & $\begin{array}{l}\text { KAKOURIS, } \\
\text { A. }\end{array}$ & 2008 & $\begin{array}{l}\text { On Initial Implementations of } \\
\text { Innovation and } \\
\text { Entrepreneurship Courses: A } \\
\text { Case Study for Undergraduates } \\
\text { at the University of Athens }\end{array}$ & $\begin{array}{c}\text { CONFERÊNCIA: 3RD EUROPEAN } \\
\text { CONFERENCE ON } \\
\text { ENTREPRENEURSHIP AND } \\
\text { INNOVATION Local: Univ } \\
\text { Winchester, Winchester, ENGLAND } \\
\text { Data: SEP 15-16, }\end{array}$ \\
\hline
\end{tabular}

Tabela 4 - As publicações e número de citações organizado por ano de publicação Fonte: Dados da Pesquisa 
Conforme a tabela 4, o artigo "The Impact of Entrepreneurship Education on Entrepreneurial Attitudes and Intention: Hysteresis and Persistence", de Fayolle e Gailly, publicado em 2015 destacou-se como o de maior impacto, com 69 citações na base Scopus e 64 na WoS. O objetivo do trabalho de Fayolle (2015) foi realizar um experimento para medir o impacto de um PEE. Foi proposto um experimento de um curso de 24 horas, ministrado em três dias para o total de 275 estudantes em nível de mestrado. Observou-se que a educação para o empreendedorismo surte mais efeito naqueles que não foram expostos anteriormente a nenhum PEE. Reconhece-se que a avaliação de programas de empreendedorismo é complexa e que a intenção empreendedora pode ser vista como o primeiro passo para a geração do comportamento empreendedor.

O segundo artigo mais referenciado, com 46 citações na base Scopus, foi "Engineering students and entrepreneurship education: Involvement, attitudes and outcomes", publicado em 2012. O objetivo foi explorar as atitudes e resultado da educação empreendedora para a carreira dos estudantes de engenharia, assim como entender o papel da autoeficácia nos cursistas. Buscou-se esclarecer sobre as características dos programas e práticas docentes que auxiliam nas melhores experiências educacionais na área do empreendedorismo. Foi aplicado um questionário de 135 itens para 501 estudantes de engenharia. O questionário foi formulado com questões das seguintes categorias: demografia, atitudes, comportamento, autoeficiência, percepções dos programas e professores. Os resultados mostraram o aumento nas habilidades empreendedoras que impactam na projeção de carreira.

Com 24 citações na base Scopus, o terceiro artigo mais referenciado foi "The Impact of Entrepreneurship Education: A Study of Iranian Students' Entrepreneurial Intentions and Opportunity Identification”, publicado em 2016. O trabalho teve como objetivo investigar o impacto dos PEE nas intenções empreendedoras, avaliando os efeitos de dois tipos de PEE, voluntário e obrigatório. Foi realizado um quase-experimento com um grupo de 275 estudantes do curso de empreendedorismo de seis universidades públicas iranianas. Não houve grupo controle. Percebeu-se que há impacto da EE nas atitudes empreendedoras e que geram efeito tanto para cursos voluntários como nos obrigatórios. No entanto, a EE dos cursos eletivos apresentaram maior propensão dos estudantes tornarem-se empreendedores.

Destacam-se ainda os artigos elaborados por Gordon (2012), que apresentou 22/17 citações. Elmuti (2012) ficou com 17 e Fayolle (2009) com 10 citações. As demais publicações apresentaram menos de 3 citações. Estes artigos sugerem que PEE desenvolvem as competências empreendedoras e geram benefícios para a projeção dos cursistas.

Os artigos analisados se subsidiaram em diversas teorias, conforme apresentado na Tabela 6.

\begin{tabular}{c|l}
\hline Teoria & \multicolumn{1}{c}{ Descrição } \\
\hline $\begin{array}{c}\text { TCP } \\
\text { Ajzen, 1985 }\end{array}$ & $\begin{array}{l}\text { Verifica as relações entre atitudes e comportamentos, levando em consideração as variáveis } \\
\text { externa e interna como influenciadoras da ação humana. A intenção é observada como uma } \\
\text { vontade de expressar um comportamento. }\end{array}$ \\
\hline $\begin{array}{c}\text { Modelo de } \\
\text { Aprendizagem } \\
\text { de Kirkpatrick } \\
\text { Alliger, 1989 }\end{array}$ & $\begin{array}{l}\text { É um modelo que apresenta quatro pressupostos para verificar a aprendizagem: 1) Verifica- } \\
\text { se reaça de quem aprende; 2) O aprendizado, medido por novos conhecimentos ou } \\
\text { níveis são interligados tende a gerar resultado positivo. }\end{array}$ \\
\hline $\begin{array}{c}\text { Ciclo de } \\
\text { Aprendizagem } \\
\text { de Kolb (1976) }\end{array}$ & $\begin{array}{l}\text { Atribui importância à experiência para a aprendizagem. Descreve o processo de } \\
\text { aprendizagem tendo como base um ciclo contínuo de quatro estágios: Experiência Concreta } \\
\text { (agir), Observação Reflexiva (refletir), Conceitualização Abstrata (conceitualizar) e } \\
\text { Experimentação Ativa (aplicar). }\end{array}$ \\
\hline
\end{tabular}

Tabela 6 - Relação das teorias e os seus respectivos conceitos.

Fonte: Elaborado pelas autoras 
Os dezoito (18) artigos de interesse analisados utilizaram diferentes aportes teóricos e apresentaram conclusões sintetizadas, conforme a Tabela 5.

\begin{tabular}{|c|c|c|c|}
\hline Artigo & Ano & Principais conclusões & Aporte Teórico \\
\hline KOLADE, O. & 2018 & $\begin{array}{l}\text { Há relação da diminuição da pobreza com a EE e geração } \\
\text { de consciência e criação de novos empreendimentos }\end{array}$ & Tobias et al., 2013 \\
\hline KOVESI, K. & 2017 & $\begin{array}{l}\text { A EE é determinante para a evolução da carreira dos } \\
\text { engenheiros. }\end{array}$ & $\begin{array}{c}\text { Tounés,2003;Fayolle, } \\
\text { 2001;Klapper, } 2005\end{array}$ \\
\hline $\begin{array}{l}\text { PEDRINI, M.; } \\
\text { LANGULLA, V.; } \\
\text { MOLTENI, M. }\end{array}$ & 2017 & $\begin{array}{l}\text { Foi observado o impacto da EE para as caractetísticas } \\
\text { psicológicas e habilidades pessoais dos egressos. }\end{array}$ & TCP \\
\hline $\begin{array}{l}\text { ARPIAINEN, R.; } \\
\text { KURCZEWSKA, A. }\end{array}$ & 2017 & $\begin{array}{l}\text { A EE tem uma natureza transformadora e pode } \\
\text { desenvolver competências voltadas para correr riscos. }\end{array}$ & $\begin{array}{l}\text { Ruohotie, 2000; Gibb, } \\
\text { 2002; Krueger, 2007, } \\
2009\end{array}$ \\
\hline $\begin{array}{l}\text { KARIMI, S.; } \\
\text { BIEMANS, H. J. A.; } \\
\text { LANS, T.; et al. }\end{array}$ & 2016 & $\begin{array}{l}\text { Foi evidenciado que os PEE devem ser melhorados e que } \\
\text { o conteúdo deve ser escolhido em função da } \\
\text { obrigatoriedade ou não dos estudantes estarem no curso. }\end{array}$ & TCP \\
\hline $\begin{array}{l}\text { DIAZ-GARCIA, C.; } \\
\text { SAEZ,-MARTINEZ, F.; } \\
\text { JIMENEZ-MORENO, J. }\end{array}$ & 2015 & $\begin{array}{l}\text { O PEE contribuiu para a escolha do } \\
\text { empreendedorismo como opção de carreira para } \\
\text { alguns cursistas. Os participantes perceberam mais } \\
\text { dificuldades ambientais do que os não participantes. }\end{array}$ & $\mathrm{TCP}$ \\
\hline $\begin{array}{l}\text { KEENA, L.; } \\
\text { SIMMONS, C. }\end{array}$ & 2015 & $\begin{array}{l}\text { EE para presos. A empregabilidade dos detentos } \\
\text { participantes do PEE foi um dos fatores que } \\
\text { evidenciaram o impacto da EE. }\end{array}$ & $\begin{array}{l}\text { Sonfield, 1994; F. S. } \\
\text { Lockwood et al., } 2006\end{array}$ \\
\hline $\begin{array}{l}\text { FAYOLLE, A.; } \\
\text { GAILLY, B. }\end{array}$ & 2015 & $\begin{array}{l}\text { A educação para o empreendedorismo surte mais } \\
\text { efeito naqueles que não foram expostos a um PEE. }\end{array}$ & Kirkpatrick e TCP \\
\hline $\begin{array}{c}\text { CARVALHO, L.; } \\
\text { COSTA, T.; MARES, P. }\end{array}$ & 2015 & $\begin{array}{l}\text { A criação e capacidade do trabalho em equipe foram } \\
\text { identificadas como fator positivo do programa de EE. }\end{array}$ & TCP \\
\hline $\begin{array}{l}\text { NASHR, B. K., } \\
\text { BOUJELBENE, Y. }\end{array}$ & 2013 & $\begin{array}{l}\text { Há relatos que os estudantes transferiram o que } \\
\text { aprenderam para as suas atuações profissionais e } \\
\text { aumentaram a intenção e perfil empreendedor. }\end{array}$ & Kirkpatrick \\
\hline $\begin{array}{l}\text { DUVAL-COUETIL, N.; } \\
\text { WHEADON, J. }\end{array}$ & 2013 & $\begin{array}{l}\text { Participantes do PEE listaram os conteúdos } \\
\text { aprendidos e o trabalho colaborativocomo importantes } \\
\text { para a projeção de carreira. O PEE ajudou-os a } \\
\text { conquistar emprego e tornar o currículo atrativo. }\end{array}$ & $\begin{array}{l}\text { Duval , 2013; } \\
\text { Sarasvathy, } 2008\end{array}$ \\
\hline $\begin{array}{c}\text { MASSI, L.; } \\
\text { GEORGIOPOULOS, M.; } \\
\text { YOUNG, C. Y.; Et. al. } \\
\end{array}$ & 2013 & $\begin{array}{l}\text { O maior benefício do PEE é o desenvolvimento da } \\
\text { personalidade profissional. As habilidades analítica e } \\
\text { de equipe são consideradas como um ganho. }\end{array}$ & $\begin{array}{l}\text { Jimenes, 2002; } \\
\text { Hunter, } 2007\end{array}$ \\
\hline $\begin{array}{l}\text { CARVALHO, M. I.; } \\
\text { SIMOES, J.; } \\
\text { SAMAGAIO, A.; et. al. } \\
\end{array}$ & 2012 & $\begin{array}{l}\text { O contexto cultural é relevante para o desenvolvimento } \\
\text { das competências empreendedoras. Sugere mais } \\
\text { atenção na formulação dos programas de EE. }\end{array}$ & $\begin{array}{l}\text { Teoria da atitude } \\
\text { versus teoria dos } \\
\text { traços }\end{array}$ \\
\hline $\begin{array}{l}\text { DUVAL-COUETIL, N.; } \\
\text { REED-RHOADS, T.; } \\
\text { HAGHIGHI, S. } \\
\end{array}$ & 2012 & $\begin{array}{l}\text { Participante do PEE concordam que aumentaram } \\
\text { habilidades e podem ampliar a perspectiva da escolha } \\
\text { de carreira. }\end{array}$ & Wei, 2005 \\
\hline $\begin{array}{c}\text { GORDON, I.; } \\
\text { HAMILTON, E.; JACK, S. }\end{array}$ & 2012 & $\begin{array}{l}\text { O estudo concluiu que a EE gerou benefícios para os } \\
\text { proprietários das empresas e desenvolveu a região em } \\
\text { que o negócio estava inserido. }\end{array}$ & Pittaway, 2007 \\
\hline $\begin{array}{l}\text { ELMUTI, D.; KHOURY, } \\
\text { G.; OMRAN, O. }\end{array}$ & 2012 & $\begin{array}{l}\text { Inclui a formação de competências sociais através do } \\
\text { PEE. }\end{array}$ & TCP \\
\hline $\begin{array}{l}\text { FAYOLLE, A.; } \\
\text { GAILLY, B. }\end{array}$ & 2009 & $\begin{array}{l}\text { Não há impacto de um PEE no curto prazo, mas após } \\
\text { seis meses é possível observá-lo }\end{array}$ & Kirkpatrick e TCP \\
\hline KAKOURIS, A. & 2008 & $\begin{array}{l}\text { Enfatiza a necessidade de formar profissionais para } \\
\text { trabalhar a EE e a carência de teorias cumulativas. }\end{array}$ & $\begin{array}{l}\text { Getzels, 1962; Kolb, } \\
1976\end{array}$ \\
\hline
\end{tabular}

Tabela 5 - Resumo dos principais temas e teorias utilizadas para verificar o impacto da EE. Fonte: Elaborado pelas autoras 
Considerando os artigos de interesse relacionados na Tabela 5, observou-se que pouco mais de 35\% dos estudos utilizaram a Teoria do Comportamento Planejado (TCP) como principal aporte teórico. Fayolle (2015) usou a TCP considerando as variações da intenção de comportamento empreendedor e seus antecedentes. Foi utilizado um questionário para medir as quatro dimensões da teoria do comportamento planejado: as atitudes a respeito do comportamento empreendedor, as normas subjetivas percebidas, controle comportamental percebido e intenção empreendedora. Já Fayolle (2009) utilizou a TCP com o objetivo de gerar indicadores de impacto da formação e das lições aprendidas.

Karimi (2016) também entende que a TCP pode ser utilizada para verificar a intenção empreendedora como antecendete do comportamento empreendedor. Tem como hipótese que a TCP previsiona efeitos sobre as normas subjetivas, o controle do comportamento percebido e atitudes em relação ao empreendedorismo. Pedrini (2017) para medir a intenção empreendedora utilizou a TCP adaptada, que inclui mais duas dimensões para análise: (a) o psicológico, evidenciado pelas características do potencial empreendedor e (b) habilidades pessoais e conhecimento do potencial empreendedor.

Elmuti (2012) abordou a TCP para observar a evolução das atitudes dos estudantes e para a "mentalidade" empreendedora. Já Carvalho (2015) ao utilizar a TCP leva em consideração as atitudes em relação: (a) ao comportamento, incluindo o reflexo da avaliação individual do comportamento; (b) Normas subjetivas que referencia ao grau em que familiares, amigos, pares e sociedade espera ou pressionam o indivíduo para se tornar empreendedor; (c) Grau de controle de comportamento percebido, definida pelo grau em que o indivíduo se sente capaz para gerar um comportamento empreendedor. Diaz-Garcia (2015) utilizou a TCP para avaliar o impacto do desenvolvimento de um PEE sobre as atitudes, intenções e crenças empreendedoras dos participantes. A proposta foi usar a TCP como uma ferramenta para investigar as variações na forma dos participantes em perceberem o meio ambiente e suas atitudes, intenções e habilidades empreendedoras. A investigação teve enfoque no final do curso e seis meses após o seu término.

O modelo de avaliação de Kirkpatrick foi utilizado como aporte teórico em aproximadamente $15 \%$ dos artigos. Este modelo tem como referência quatro níveis no trabalho de avaliação que são interligados: (1) Reação, que se refere às reações apresentadas pelos participantes ao fim do programa; (2) Aprendendo, relaciona em que medida os objetivos de aprendizagem foram satisfeitos; (3) Comportamento, refere-se à identificação de mudanças comportamentais após o treinamento; (4) Resultados, relaciona-se com os benefícios para o indivíduo/empresa após a participação de um programa de aprendizagem.

Fayolle (2009) avalia a aprendizagem, que se refere ao nível 2 do modelo de Kirkpatrick. Este nível tem relação com o melhoramento de técnicas e atitudes relacionadas à aprendizagem. Nashr (2013) centrou a sua pesquisa em dois aspectos: (a) Perfil empreendedor dos participantes; (b) Carreira dos participantes examinando como eles transferiram seus conhecimentos para seus trabalhos profissionais. Neste estudo percebe-se que foi avaliado até o quarto nível de Kirkpatrick que se refere a resultados. Fayolle (2015) apresenta o seu estudo para testar o impacto de um PEE nas intenções empreendedoras. $O$ autor afirma que comportamento e resultado são os níveis do modelo de Kirkpatrick menos estudados.

Avaliar o impacto da EE tem sido um dos principais desafios dos pesquisadores. Entre os artigos analisados e apresentados na Tabela 5, observou-se os resultados de pesquisa que procuravam medir o impacto de programas de educação empreendedora foram publicados em 2008. O artigo descreveu o projeto pedagógico de um curso de EE e suas implicações para o desenvolvimento de competências empreendedoras dos estudantes. A metodologia utilizada 
foi um quase-experimento com amostra de 222 alunos jovens. Os autores defendem a necessidade de formar profissionais para trabalhar a EE e identificam lacunas nas teorias do campo do empreendedorismo. O curso ofertado nesta pesquisa não gerou mudanças significativas nos fatores psicológicos dos estudantes, embora tenha mudado a visão dos estudantes em relação ao conhecimento sobre empreendedorismo. A mudança da visão média dos estudantes sobre o empreendedorismo foi atribuída à vivência experiencial dos cursistas e considerada como irrelevante para uma aprendizagem transformadora (KAKOURIS, 2008).

Em seguida, Allan Fayolle (2009), reconhecido pesquisador na área de educação empreendedora da EMLYON Business School, em Lyon, publicou artigo, em co-autoria com Benoit Gailly, em que procurava medir a intensidade do impacto de um PEE sobre a intenção empreendedora dos participantes. Os autores se utilizaram da teoria de Kirkpatrick, referência há mais de 30 anos para medir a eficácia dos programas de treinamento, e a teoria do comportamento planejado (TCP), a fim de observar a intenção de empreender dos cursistas. Os achados evidenciam que não há impacto de um PEE no curto prazo, mas após seis meses é possível observar diferença de atitudes, na percepção de normas subjetivas e na percepção do controle comportamental. Não foi demonstrado link entre o nível de intenção empreendedora com a presença de parentes empreendedores.

O interesse em avaliar a contribuição de PEE para os egressos vem aumentando, o que se reflete no número de artigos publicados em 2012. Neste ano foram publicados 4 artigos (GORDON, 2012; ISLAM, 2012; ELMUTI, 2012; CARVALHO, 2012). Ian Gordon, Eleanor Hamilton \& Sarah Jack (2012) tratou da aprendizagem das Micro e Pequenas Empresas (ME), identificando o desenvolvimento de competência para articular redes de contatos como elemento central para a construção de uma formação empreendedora. As pessoas funcionam como um "catalisador" na criação de mudanças. A informalidade nos programas de EE foi mencionada como um dos fatores que contribuem para o aumento da rede de contatos e vai além do período de duração do PEE. O estudo concluiu que a EE gerou benefícios para os proprietários das empresas e desenvolveu a região em que o negócio estava inserido.

Para Duval-Couetil (2012), o grupo participante do PEE concorda que a EE aumentou suas habilidades e pode ampliar a perspectiva da escolha de carreira. Os estudantes apresentaram mais propensão para aprendizagem que envolve habilidades práticas, como análise de mercado, comunicação comercial e outras. Os participantes que já tinham estudado empreendedorismo demonstraram mais autoeficácia.

Elmuti (2012) examina o impacto de treinamento voltado para aperfeiçoar habilidades empreendedoras, observando a interelação deste treinamento com a eficácia dos empreendimentos. Foi realizada uma pesquisa com 500 pessoas que tinham interesses em iniciar um negócio. Foram aplicados questionários divididos em quatro partes. Um conjunto de variáveis foram usadas para medir a eficácia organizacional como: desempenho (rentabilidade, vendas, ROI e participação de mercado); adaptabilidade (flexibilidade, vontade de mudar, adotar e inovar) e satisfação (alcançar os objetivos da empresa e alcançar as necessidades de funcionários e empresários). Observou-se impacto positivo da EE nos cursistas, como resultado das interações sociais e reflexões que estimulam a criatividade. Os participantes dos PEE foram impactados, principalmente nas competências sociais.

Carvalho (2012) examinou a influência dos PEE sobre o desejo dos jovens trabalharem por conta própria e desenvolverem competências empreendedoras. $\mathrm{O}$ estudo foi dirigido ao ensino secundário e obteve 182 respostas. Observou-se indícios de impacto positivo da educação empreendedora nos cursistas, contudo, não houve evidencias da escolha de trabalhos por conta própria na maioria dos respondentes. Eles preferiram a afiliação como 
funcionários. Os achados destacam a relevância do contexto cultural para o desenvolvimento das competências empreendedoras e sugere mais atenção na formulação dos PEE.

Outro estudo demonstou que há impacto positivo da EE na carreira dos estudantes de pós-graduação, em nível de mestrado. Há relatos que os estudantes aplicaram o que aprenderam no seu exercício profissional e aumentaram a intenção para empreender. Uma das métricas utilizadas pelo autor para medir os efeitos da $\mathrm{EE}$ foi o desemprego. No fim do programa o índice de desemprego diminuiu 31,1\%. O autor utilizou o modelo de Kirkpatrick (1959) (NASR, 2013). Duval (2013), mostrou que os participantes do PEE listaram os conteúdos aprendidos como importantes para a projeção de carreira, contudo, percebem que aprender a resolver problemas é mais válido do que o conteúdo específico. Os participantes do PEE afirmaram que a EE os ajudou a conquistar um emprego e tornar o currículo mais atrativo. A mentalidade empreendedora, incluindo o aumento da autoeficácia, predisposição para abrir um negócio e escolha de carreira empreendedora, também, foram apresentadas como uma das características desenvolvida pela EE.

A pesquisa desenvolvida por Massi (2013) com estudantes de engenharia e matemática que tenham declarado interesse pelo empreendedorismo concluiu que o desenvolvimento de habilidade analítica e de trabalho em equipe foram consideradas pelos egressos como contribuições centrais do programa. Há percepção de benefícios pessoais e profissionais positivos e contribuição para o ganho de competências empreendedoras como a autoconfiança e capacidade para construir e manter redes de relacionamento (Massi, 2013).

Outro estudo apresentado teve como público alvo detentos de uma prisão de segurança máxima e os resultados foram positivos. Foi realizado um estudo de caso com entrevistas analisadas através do método comparativo constante. Não houve grupo de comparação. A empregabilidade dos detentos participantes do PEE foi um dos fatores que evidenciaram o impacto da EE. A perseverança foi citada como essencial para a geração da empregabilidade. Diferente de outros presos, os participantes do PEE demonstraram ao final do curso maior confiança, ousadia e persistência. O pesquisador mediu a persistência dos cursista através da persistência das palavras. Ou seja, os participantes, após aplicado um estudo de caso, explicaram como foi entendido o poder da persistência na obtenção de emprego remunerado. Também, foram percebidas mudanças no desenvolvimento pessoal e cognitivo do grupo. Um exemplo apresentado foi o hábito de ler jornal. Um participante declarou que se surpreendeu ao perceber que depois do curso tinha criado o hábito de ler jornal. Os participantes entenderam e aplicaram os conceitos desenvolvidos no PEE (KEENA, 2015).

Dias-Garcia (2015) apresentou artigo em que comparou dois grupos de estudantes. Foi aplicado um curso para um grupo de 170 estudantes que foi comparado com um grupo controle. Aplicou-se um questionário no final do curso e um de acompanhamento seis meses depois do término do curso. Os autores apresentaram como limitação do estudo o fato de não terem aplicado um questionário no início do curso. Os participantes dos PEE perceberam mais dificuldades ambientais. As dificuldades ambientais são reconhecidas como a percepção de dificuldades enfrentadas no ambiente ou percepção de risco. Os cursistas desenvolveram competências como autoeficácia, atitudes e intenção empreendedora, o que contribuiu para a escolha do empreendedorismo como opção de carreira por alguns.

Para Fayolle (2015) a educação para o empreendedorismo surte mais efeito naqueles que não foram expostos a nenhum programa de EEP anteriormente. $\mathrm{O}$ autor realizou um experimento de um curso de 24 horas, ministrado em três dias para o total de 275 estudantes mestrandos, com a finalidade de medir o impacto de um PEE. Segundo Carvalho (2015), a intenção empreendedora pode ser avaliada através de duas dimensões: dimensão pessoal e 
dimensão do potencial empreendedor. Foram aplicados questionários para os estudantes de um programa de empreededorismo, cuja amostra foi de 28 estudantes de ensino médio. Os dados coletados foram analisados considerando estatística descritiva e análise de conteúdo. Os fatores internos (personalidade) e externos (ambiental ou contextual) são mencionados como uma maneira de verificar a intenção empreendedora. A criação e capacidade do trabalho em equipe são um dos fatores positivos do PEE. Além disso, concluiu-se que o PEE influi no projeto de vida dos jovens, envolvendo dimensões pessoais e profissionais.

A pesquisa realizada por Karimi (2016), com estudantes iranianos, sugere que a EE tem efeito tanto para cursos voluntários como obrigatórios. No entanto, a EE dos cursos eletivos apresenta mais propensão dos estudantes tornarem-se empreendedores. $\mathrm{O}$ autor recomenta que para disciplinas obrigatórias que tratem de empreendedorismo é indicada a utilização de um programa de conscientização da educação empreendedora, que enfatize a possibilidade do empreendedorismo como uma opção de carreira. Em curso eletivos, o autor sugere que o foco seja a criação de startups. Os cursos ou disciplinas que buscam desenvolver competências empreendedoras podem ser indicados tanto como obrigatórios como eletivos. A pesquisa mostrou que há impacto da EE nas atitudes empreendedoras que foi verificado através da TCP com um "olhar" sobre a autoeficácia (KARIMI, 2016).

No ano de 2017 foram apresentados 3 artigos sobre a temática em questão. O estudo realizado por Kovesi (2017), com estudantes franceses de pós-graduação em engenharia, concluiu que a EE é determinante para a evolução da carreira e do futuro dos engenheiros. A criatividade e o pensamento interdisciplinar são apresentados como competências importantes no desenvolvimento da EE. Os contextos culturais também devem ser levados em consideração, visto que apresentam características como medo de falhar na criação de um novo empreendimento e consciência de risco (KOVESI, 2017). Foi observado o impacto da EE sobre as caractetísticas psicológicas e habilidades pessoais, tendo como base a TCP. As competências empreendedoras como autoeficácia, correr riscos calculado, criatividade, conhecimento e rede de contato foram percebidas após a exposição do PEE (PEDRINI, 2017).

O estudo realizado por Arpiainen (2017) sugere que a EE tem uma natureza transformadora e pode desenvolver competências direcionadas para correr riscos. Há benefício do trabalho em equipe para elevar a capacidade de correr risco e lidar com a incerteza (ARPIAINEN, 2017). Observou-se que houve impacto do PEE na carreira dos egressos, sendo ela empreendedora ou outra. O impacto foi avaliado comparando decisões de carreiras dos participantes do programa com pessoas não aceitas no programa. Ao medir o impacto do empreendedorismo em pessoas já expostas à temática e outra não exposta, foi percebido que os PEE parecem ser mais eficientes em pessoas que não participaram de outras iniciativas que objetivavam desenvolver competências empreendedoras antes do curso. A experiência de empreendedorismo anterior e a pontuação de uma entrevista foram utilizadas para medir a predisposição dos cursistas (LYONS, 2018).

O estudo publicado mais recente identificou uma relação da diminuição da pobreza com a EE. Foi explorado o caso da intervenção EE na Nigéria, cidade de Maiduguri, que é uma área caracterizada pelas altas taxas de desemprego e violência terrorista, entre outros fatores que caracterizam ampla pobreza. A mudança positiva da mentalidade, desenvolvimento, geração de consciência e criação de novos empreendimentos estão relacionados ao desenvolvimento de PEE. É ressaltada a importância de um currículo de EE dinâmico, a necessidade de capacitação na área e a expansão para níveis diferentes do ensino superior (KOLADE, 2018). 


\section{Considerações Finais}

Este estudo identificou os principais autores, publicações, teorias e abordagens que tratam sobre a contribuição da educação empreendedora para os egressos dos cursos. O período compreendido entre 2012 a 2015 concentrou o maior número de publicações sobre o tema. Foi realizada a leitura de 434 registros das bases de dados, contudo, somente 18 artigos estavam relacionados diretamente ao tema contribuição da EE para os cursistas.

Os artigos de maior repercussão, publicados em 2015 e 2012, evidenciam que a educação para o empreendedorismo surte mais efeito entre pessoas que não foram expostas a nenhum programa de EE anteriormente (Fayolle, 2015). A pesquisa que explorou as atitudes e os resultados da educação empreendedora para a carreira dos estudantes de engenharia evidenciou melhoria nas habilidades empreendedoras que impactam a carreira profissional (DUVAL-COUETIL, 2012).

Os artigos analisados nesta bibliometria abordaram programas de educação empreendedora destinados a diversos públicos, como: estudantes de mestrado, estudantes de graduação, estudantes de ensino secundário, microempresários e presidiários. Os estudos utilizaram abordagens metodológicas distintas, prevalecendo o estudo de caso. Os achados apresentam a necessidade de formar profissionais para trabalhar a educação empreendedora e identificaram lacunas nas teorias do campo do empreendedorismo. Tendo profissionais qualificados a EE tende a ser desenvolvida nos cursistas de forma mais eficaz, aumentando a probabilidade de serem percebidos efeitos positivos dos PEE.

A temática mais explorada nos artigos analisados está relacionada à medição do impacto de um PEE sobre a intenção empreendedora. Para esta finalidade os autores se utilizaram da Teoria do Comportamento Planejado (TCP). Observou-se que pouco mais de $35 \%$ dos estudos utilizaram a Teoria do Comportamento Planejado (TCP) como principal aporte teórico. Já o modelo de avaliação de Kirkpatrick foi utilizado em aproximadamente $15 \%$ dos artigos.

A TCP foi utilizada com os seguintes objetivos: (a) verificação da intenção empreendedora que serve como observância para o comportamento empreendedor; (b) para gerar indicadores de impacto da formação e das lições aprendidas; (c) visando o direcionamento para a "mentalidade" empreendedora; (d) como uma ferramenta para investigar as variações na forma dos participantes dos cursos em perceberem o meio ambiente e suas atitudes, intenções e habilidades empreendedoras. Foram utilizadas as quatro dimensões da TCP na maior parte dos estudos, contudo, 1 artigo incluiu duas dimensões novas para análise: (a) o psicológico, evidenciado pelas características do potencial empreendedor e (b) habilidades pessoais e conhecimento do potencial empreendedor.

O modelo de avaliação de Kirkpatrick foi utilizado para avaliar a aprendizagem. Um artigo explorou até o nível dois do modelo, que tem por referência quatro níveis. Foi observado o melhoramento de técnicas e atitudes relacionadas à aprendizagem. O modelo foi utilizado para pesquisar os seguintes aspectos: (a) Perfil empreendedor dos participantes; (b) Carreira dos participantes, examinando como eles transferiram seus conhecimentos para seus trabalhos profissionais; (c) para medir o impacto de um programa de educação empreendedora através das intenções empreendedoras.

Como um apanhado geral, os achados dos artigos pesquisados sugerem que programas de educação empreendedora ampliam a perspectiva da escolha de carreira, ajudam na conquista de um emprego, tornam o currículo atrativo e contribuem para o desenvolvimento de competências sociais e psicológicas. 
Referências Bibliográficas

ARPIAINEN, R.; KURCZEWSKA, A. Learning risk-taking and coping with uncertainty through experiential, team-based entrepreneurship education. Industry and Higher Education, v. 31, n. 3, 1 jun. 2017, p. 143-155, 2017.

ALLIGER, G. M.; JANAK, E. A. Kirkpatrick's Levels of Training Criteria: Thirty Years Later. Personnel Psychology 42, 331-342, 1989.

AJZEN, I. From intention to actions: A theory of planned behavior. In J. Kuhl, \& J. Beckman (Eds.), Action control: From cognition to behavior, p. 11-39. New York: Springer-Verlag., 1985.

BARDIN, L. Análise de conteúdo. Lisboa: Edições 70, 2006.

CARVALHO, M. I.; SIMOES, J.; SAMAGAIO, A.; et. al. Enterprise Potential of Portuguese Students Fostered by an Entrepreneurship Education Program. Asee Annual Conference And Exposition, Conference Proceedings, 2012.

CARVALHO, L.; COSTA, T.; MARES, P. A success story in a partnership programme for entrepreneurship education: Outlook of students perceptions towards entrepreneurship. International Journal Of Management In Education. V.9, E. 4, 2015, p. 444-465, 2015.

CARREIRA. Dicionário online do Aurélio, 26 jun. 2017. Disponível em: 〈https://dicionariodoaurelio.com/eficaz〉. Acesso em: 16 Fev. 2018.

CHANLAT, J. F. Quais carreiras e para qual sociedade? Revista Administração de empresas., São Paulo, v. 36, n. 1, p. 13-20, Mar. 1996.

CALLANAM, G. A.; ZIMMERMAM, M. To Be or Not To Be an Entrepreneur: Applying a Normative Model to Career Decisions. Journal of Career Development. V. 43, p.447-461, 2016.

DIAZ-GARCIA, C.; SAEZ,-MARTINEZ, F.; JIMENEZ-MORENO, J. Assessing the impact of the "Entrepreneurs" education programme on participants' entrepreneurial intentions. Rusc-universities and Knowledge Society Journal V.: 12 n.3 p. 17-31, 2015. DUVAL-COUETIL, N.; WHEADON, J. The Value of Entrepreneurship to Recent Engineering Graduates: A Qualitative Perspective. Conferência: 43rd Annual Frontiers In Education Conference (fie), Univ Oklahoma, Coll Engn, Oklahoma City, Oct 23-25, 2013.

DUVAL-COUETIL, N.; KISENWETHER, TRANQUILO, J.; WHEADON, J. Exploring the alignment of entrepreneurship education with ABET accreditation criteria, in National Collegiate Inventors and Innovators Alliance Open Conference, 2013.

DUVAL-COUETIL, N.; REED-RHOADS, T.; HAGHIGHI, S. Engineering students and entrepreneurship education: Involvement, attitudes and outcomes. International Journal of Engineering Education Vol. 28, No. 2, pp. 425-435, 2012.

DRUCKER, P. F. Inovação e espírito empreendedor: práticas e princípios. São Paulo: Pioneira, 1998.

ELMUTI, D.; KHOURY, G.; OMRAN, O. Does entrepreneurship education have a role in developing entrepreneurial skills and ventures' effectiveness? Journal of Entrepreneurship Education. V. 15, p. 83-98, 2012.

FAYOLLE, A. D'une approche typologique de l'entrepreneuriat chez les ingénieurs à la reconstruction d'itinéraires d'ingénieurs entrepreneurs, Revue de l'entrepreneuriat, 1, p. 77-97, 2001.

FAYOLLE, A.; GAILLY, B. Évaluation d'une formation en entrepreneuriat: Prédispositions et impact sur l'intention d'entreprendre. Management. V. 12, p. 175-203, 2009

FAYOLLE, A. Personal views on the future of entrepreneurship education, Entrepreneurship \& Regional Development: An International Journal, 25:7-8, 692-701, 2013. FAYOLLE, A; GAILLY, B. The impact of entrepreneurship education on 
entrepreneurial attitudes and intention: Hysteresis and persistence. Journal of Small Business Management 53 (1), 75-93, 2015.

FILION, L. J. Entendendo os intraempreendedores como visionistas. Revista de Negócios, v. 9, n. 2, p. 65-80, 2004.

GETZELS, J. Creativity and Intelligence, Routledge, London, 1962.

GIBB, A. In pursuit of a new "enterprise" and "entrepreneurship" paradigm for learning: creative destruction, new values, new ways of doing things and new combinations of knowledge. International Journal of Management. Review 4: 233-269, 2002.

GORDON, I.; HAMILTON, E.; JACK, S. A study of a university-led entrepreneurship education programme for small business owner/managers. Entrepreneurship and Regional Development. V. 24, Issue 9-10, December 2012, P. 767-805, 2012.

HUNTER, A,; LAURSEN, S. L.; SEYMOUR, E. Becoming a scientist: The role of undergraduate research in students' cognitive, personal, and professional development. Science Education 91(1), 36-74, 2007.

MARCELO, J. F.; HAYASHI, M. C. P. I. Estudo bibliométrico sobre a produção científica da área da sociologia da ciência. Informação \& Informação. V. 18, n. 3, p. 138-153, Set, 2013. MARIANO, S.; MAYER, V. F. Empreendedorismo: fundamentos e técnicas para a criatividade. Rio de Janeiro: LTC, 2012.

MOBERG, K. Assessing the Impact of Entrepreneurship Education. 2014. 238f. Tese de doutorado. Copenhagen Business School. Dinamarca, 2014.

MORAES, J. et al. Uma proposição Espistêmica quadripolar do constructo empreendedorismo. REGE Revista de Gestão, São Paulo, v. 22, n. 4, p. 545-564, feb. 2016.

ISLAM, R. Engineering students and entrepreneurship education: Involvement, attitudes and outcomes. Asee Annual Conference and Exposition, Conference Proceedings, 2012.

JIMENEZ, M.; PALOMERA, R.; TOLEDO, M. Undergraduate research and co-op education: A winning combination. Proceeding. 32nd ASEE/IEEE Frontiers in Education Conference, p. 2-6, Nov. 2002.

KAKOURIS, A. On Initial Implementations of Innovation and Entrepreneurship Courses: A Case Study for Undergraduates at the University of Athens. Conferência: 3rd European Conference on Entrepreneurship and Innovation Local: Univ Winchester, Winchester, Inglaterra, p. 15-16. Sep, 2008.

KARIMI, S.; BIEMANS, H. J. A.; LANS, T.; et al. The Impact of Entrepreneurship Education: A Study of Iranian Students' Entrepreneurial Intentions and Opportunity Identification. Jornal Of Small Business Management V. 54 v: 1 p. 187-209, 2016.

KEENA, L.; SIMMONS, C. Rethink, Reform, Reenter: An Entrepreneurial Approach to Prison Programming. International Journal Of Offender Therapy And Comparative $\begin{array}{lllll}\text { Criminology } & \text { v: } 59 & \text { n. } 8 & \text { p: } 837-854\end{array}$

KLAPPER, R. Training entrepreneurship at a French grande école: The Projet Entreprendre at the ESC Rouen, Journal of European Industrial Training, 29, p. 678-693, 2005.

KOLADE, O. Venturing under fire: Entrepreneurship education, venture creation, and poverty reduction in conflict-ridden Maiduguri, Nigeria. Education and Training, v. 22, 2018.

KOLB, D.A. The learning style inventory: Technical manual, McBer, Boston, 1976.

KOVESI, K. The changing face of entrepreneurship education for engineering students in France. Conferência: 8th Ieee Global Engineering Education Conference (educon) Local: Atenas, Grécia, Abr. p. 25-28, 2017. 
KRUEGER, NF. The Microfoundations of Entrepreneurial Learning and Education. The Handbook of University Wide. Entrepreneurship. Cheltenham, UK: Edward Elgar, 2009.

KRUEGER, NF. What lies beneath? The experiential essence of entrepreneurial thinking. Entrepreneurship Theory and Practice 31(1): 123, 2007.

LANDSTRÖM.,H., HARIRCHI, G., ASTRÖM, F. Entrepreneurship: exploring the knowledge base, Research Policy, 41 (7), 1154-1181, 2012.

LOCKWOOD, F. S., TEASLEY, R.; CARLAND, J. C.; CARLAND, J. W. An examination of the power of the dark side of entrepreneurship, 2006.

LOPES, R. M. A. Ensino de Empreendedorismo no Brasil: Panorama, tendências e melhores práticas. $1^{\text {a }}$ Edição . Rio de Janeiro: Alta Books. Ed., 2017.

MASSI, L.; GEORGIOPOULOS, M.; YOUNG, C. Y.; Et. al. Internships and Undergraduate Research: Impact, Support, and Institutionalization of an NSF S-STEM Program through Partnerships with Industry and Funding from Federal and Local Workforce Agencies. Conferência: Asee Annual Conference. Atlanta, Jun, p. 23-26, 2013.

NASHR, B. K., BOUJELBENE, Y. Assessing the impact of entrepreneurship education. conferência: 2nd world conference on business, economics and management (bem). local: antalya, turkey data: apr 25-28, 2013.

PEDRINI, M.; LANGULLA, V.; MOLTENI, M. Do entrepreneurial education programs impact the antecedents of entrepreneurial intention? An analysis of an entrepreneurship MBA in Ghana. Journal Of Enterprising Communities-people And Places Of Global Economy, V.: 11 n.3 Ed. especial: SI p. 373-392, 2017.

PITAWAY, L.; COPE, J. Entrepreneurship education: A systematic review of the evidence. International Small Business Journal 25, no. 5: 479-507, 2007.

RUOHOTIE, P. Conative constructs in learning. In: Pintrich PR and Ruohotie P (eds), Conative Constructs and Self-regulated Learning. Research Centre for Vocational Education H“ameenlinna, Finland. Saarij”arvi, Finland: Saarij”arven Offset Oy, 2000.

ROSENDAHL-HUBER et al., The Effects of Early Entrepreneurship Education: Evidence from a Randomized Field Experiment. Discussion Paper Series, IZA DP V. 6512, 2012.

SARASVATHY, S. Effectuation: Elements of Entrepreneurial Expertise. London: Edward Elgar Publishing, pp. 1-392, 2008.

SONFIELD, M.; BARBATO, R. Testing prison inmates for entrepreneurial aptitude in comparison to other groups. Journal of Small Business Strategy, 2, 45-51, 1994.

SONFIELD, M ; LUSSIER, R.; BARBATO, R. The entrepreneurial aptitude of prison inmates and the potential benefit of self-employment training program. Academy of Entrepreneurship Journal, V. 7, p. 85-94, 2001.

TAMS, S. \& Arthur, M.B. The boundaryless career. In: Greenhouse, J. H. \& Callanan, G. A. Encyclopedia of Career Development: V.1. London, U. K.: Sage Publications Ltd, p. 44-49. TOBIAS, J.; MAIR, J., BARBOSA-LEIKER, C. Toward a theory of transformative entrepreneuring: poverty reduction and conflict resolution in Rwanda's entrepreneurial coffee sector", Journal of Business Venturing, Vol. 28 No. 6, pp. 728-742, 2013.

TOUNÉS, A. Un cadre d'analyse de l'enseignement de l'entrepreneuriat en France. Agence universitaire de la francophonie, Réseau entrepreneuriat, 2003.

WEI, J. Engineering education for a post-industrial world. Technology in Society. V. 27. pp. 123-132, 2005.

ZHAO, H., HILLS, G.E., SEIBERT, S. The mediating role of self-efficacy in the development of entrepreneurial intentions. Journal of Applied Psychology, 90, 6, p. 1265-1272, 2005. 